Article

\title{
A Non-Uniformly Under-Sampled Blade Tip-Timing Signal Reconstruction Method for Blade Vibration Monitoring
}

\author{
Zheng Hu ${ }^{1, \dagger}$, Jun Lin ${ }^{1, \dagger, *}$, Zhong-Sheng Chen ${ }^{1}$, Yong-Min Yang ${ }^{1}$ and Xue-Jun Li ${ }^{2}$ \\ 1 Science and Technology on Integrated Logistics Support Laboratory, National University of \\ Defense Technology, Changsha 410073, China; E-Mails: zhenghu@nudt.edu.cn (Z.H.); \\ czs_study@sina.com (Z.-S.C.); yangyongmin@163.com (Y-M.Y.)
}

2 Hunan Province Key Laboratory of Health Maintenance of Mechanical Equipment, Hunan University of Science and Technology, Xiangtan 411100, China; E-Mail: hnkjdxlxj@163.com

$\dagger$ These authors contributed equally to this work.

* Author to whom correspondence should be addressed; E-Mail: linj@163.com; Tel.: +86-159-7416-7509.

Academic Editor: Vittorio M.N. Passaro

Received: 1 December 2014 / Accepted: 16 January 2015 / Published: 22 January 2015

\begin{abstract}
High-speed blades are often prone to fatigue due to severe blade vibrations. In particular, synchronous vibrations can cause irreversible damages to the blade. Blade tip-timing methods (BTT) have become a promising way to monitor blade vibrations. However, synchronous vibrations are unsuitably monitored by uniform BTT sampling. Therefore, non-equally mounted probes have been used, which will result in the non-uniformity of the sampling signal. Since under-sampling is an intrinsic drawback of BTT methods, how to analyze non-uniformly under-sampled BTT signals is a big challenge. In this paper, a novel reconstruction method for non-uniformly under-sampled BTT data is presented. The method is based on the periodically non-uniform sampling theorem. Firstly, a mathematical model of a non-uniform BTT sampling process is built. It can be treated as the sum of certain uniform sample streams. For each stream, an interpolating function is required to prevent aliasing in the reconstructed signal. Secondly, simultaneous equations of all interpolating functions in each sub-band are built and corresponding solutions are ultimately derived to remove unwanted replicas of the original signal caused by the sampling, which may overlay the original signal. In the end, numerical simulations and experiments are carried out to validate the feasibility of the proposed method. The results demonstrate the accuracy of the reconstructed signal depends on the
\end{abstract}


sampling frequency, the blade vibration frequency, the blade vibration bandwidth, the probe static offset and the number of samples. In practice, both types of blade vibration signals can be particularly reconstructed by non-uniform BTT data acquired from only two probes.

Keywords: blade tip-timing; non-uniformly sampled signal; under-sampled signal reconstruction; on-line vibration; band-pass sampling

\section{Introduction}

High cycle fatigue (HCF) is a common failure mode of high-speed rotating blades in turbo-machinery, such as engine compressor and turbine blades [1]. It often induces blade cracks during the operation, and even catastrophic accidents. Generally speaking, blade vibrations, including synchronous and asynchronous vibrations, are major reasons behind HCF. In particular, when the blade frequency of synchronous vibrations is an integer multiple of the rotating frequency of the bladed-disk, the vibration amplitude of one blade will increase rapidly, which will cause irreversible damage to the blade. Thus nowadays it is urgent to carry out on-line blade vibration monitoring [2-18]. From vibration measurements, stresses induced in the blades may be determined. Action can then be taken to avoid harmful stresses. In this way, it is possible to predict the durability and the life of blades under operating conditions.

Blade tip-timing (BTT) methods have been proposed for non-contact blade vibration monitoring for many years [3-6]. Their outstanding advantages over conventional strain gages are that they are non-contact and can online monitor all-blade vibrations simultaneously. Classical BTT sensors include capacitive, optical-fiber, microwave probes. Among these, optical probes have the highest resolution, so they have been used widely [4,6]. Methods for analyzing BTT data have been developed for a number of applications, such as modal parameter identification of mistuned bladed disks [13,19]. However, since the sampling frequency of BTT methods is determined by the rotation speed and the number of BTT probes, it is always lower than the Nyquist frequency, i.e., twice the maximum frequency of the blade vibration signal. Therefore, the vibration signals collected by BTT methods are always well under-sampled according to the Shannon sampling theorem [20].

Up to now, many studies have been done on monitoring asynchronous vibrations using equally-mounted BTT probes. Zielinski et al. [5] used six equally-mounted probes to obtain conclusive vibration frequencies and amplitudes. Garrido et al. [8,9] proposed an autoregressive method to obtain modal parameters based on equally-mounted BTT probes. Beauseroy et al. [18] proposed a new method to analyze multicomponent blade vibrational signals based on groups of regularly spaced optical sensors. However, in these methods it was difficult to avoid aliasing due to under-sampling. In order to overcome this problem, Bendali et al. [13] proposed alternative methods to reconstruct uniformly under-sampled BTT signals for asynchronous vibrations based on the Shannon sampling theorem. Furthermore, Chen et al. [14,15] improved the work of Bendali and proposed a novel reconstruction algorithm by combining the Shannon sampling theorem and wavelet packet transformation. In this way, multiple accurate features of asynchronous vibrations are extracted in both the time and frequency domains. 
Compared with asynchronous vibrations, synchronous ones are more dangerous to the blades. Unfortunately, the conventional uniform sampling method will be invalid in measuring such vibrations. The reason is that blade vibration frequencies will be an integer multiple of the sampling rate. Assuming that the bladed-disk keeps running at a constant speed, the blade tips will have nominally the same displacement every time they pass the probes. That is to say, the blade tip displacements are essentially repeated over multiple rotations. Therefore, it is nearly impossible to extract true vibration characteristics from the sampling BTT signals. In order to solve this problem, non-equally mounted BTT probes have to be used, instead of equally-mounted ones. The big advantage is that they can be used to measure asynchronous and synchronous blade vibrations simultaneously. However, how to analyze non-uniformly under-sampled BTT signals is a big challenge, including irregular vibration data and frequency aliasing. According to the literature, little work has been done on reconstructing non-uniformly under-sampled BTT signals. To address this knowledge gap this paper will therefore explore a novel method for non-uniformly under-sampled BTT data reconstruction, which should be of great use in blade vibration monitoring.

The remainder of this paper is organized as follows: firstly, the difficulties of monitoring synchronous vibrations are stated in Section 2. In Section 3, a mathematic model of a non-uniform BTT sampling process is built and blade vibration displacements are derived. Then a reconstruction algorithm is proposed in Section 4 based on the periodically non-uniform band-pass sampling theorem. In Section 5 and Section 6, numerical simulations and experimental tests are carried out to validate the feasibility of the proposed method. Finally, some major conclusions are summarized in Section 7.

\section{Problem Statement}

As shown in Figure 1, optical-fiber probes are embedded non-equally into a stationary casing around a bladed disk. Then the times at which the blade tips pass each probe can be measured. It can be seen that the angles between these sensors are different, so it is a typical non-uniform BTT sampling process.

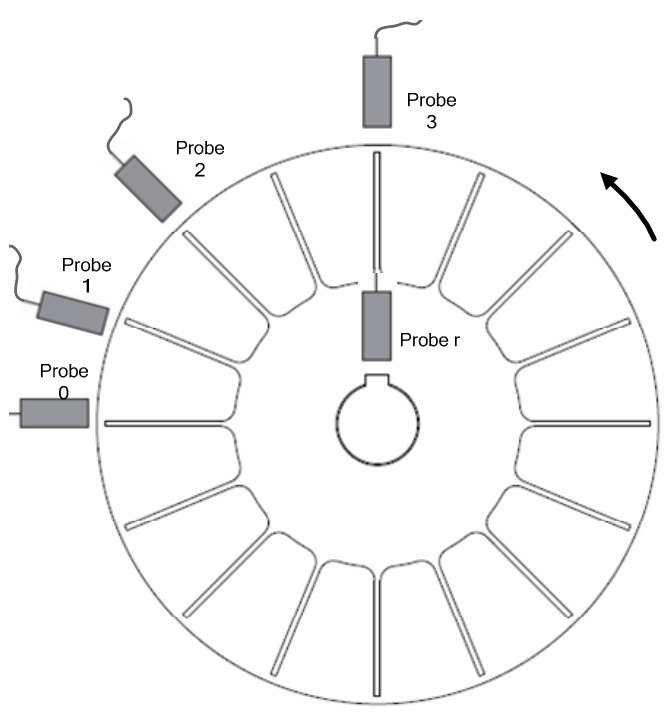

Figure 1. Schematic of the non-uniform BTT sampling. 
Suppose that the rotating period of a rotor is $T_{r}$ and the blade vibration period is $T_{r} / 2$. For a uniform sampling, eight probes are mounted equally to monitor blade vibrations. Each probe associates with a time-series of blade tip displacements, called periodically uniform sample stream. As Figure 2a shows, eight probes are numbered sequentially, starting with one for the first probe. Obviously, the time interval between successive samples is constant, i.e., $t_{3}-t_{2}=t_{2}-t_{1}$. However, it is also easily found that one or more probes see the same point on the vibration waveform over multiple rotations, such as probes 1 and probe 5, probes 2 and probe 6, and so on. They record the same displacements every time the blade tips pass them. This phenomenon has two adverse effects. The first one is that probes 5-8 are duplicated for redundancy, which is a waste of probes. The second is that the number of available displacements is reduced from 8 to 4 . This makes the analysis of the data become more difficult. In particular, when the blade vibration frequency is an integer multiple of the sampling rate, the number of available displacements can be reduced to 1 . This decrease determines that uniform sampling is unsuitable to measure synchronous vibrations.

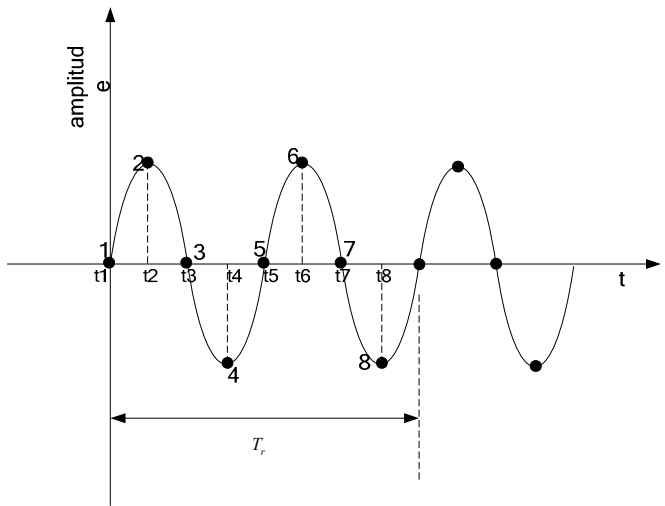

(a)

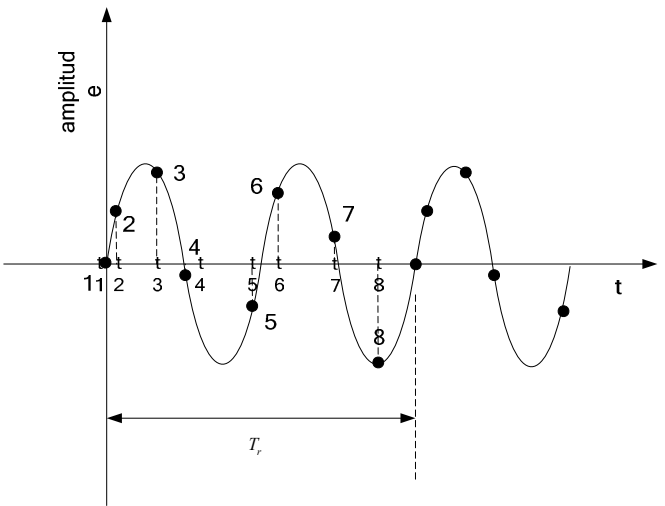

(b)

Figure 2. (a) The uniform sampling; (b) The non-uniform sampling.

Conversely, an alternative non-uniform sampling occurs by using eight non-equally mounted probes. As shown in Figure 2b, it is also a sum of eight periodically uniform sample streams. However, in this way, the time interval between successive samples is not constant for all samples, i.e., $t_{3}-t_{2} \neq t_{2}-t_{1}$. Eight points on the vibration waveform are different from each other, so that enough available information can be provided to monitor synchronous vibrations. In fact, it allows measurement of both types of blade vibrations with a reduced number of probes.

Except for the above drawback of uniform sampling, one should attach importance to another fact. That is, in practice, probes are hardly equally spaced in the outer casing due to minimal manufacturing tolerances. Reference [18] has investigated the frequency spectrum of such uniform BTT data. The results indicate that in this way, BTT sampling acts like non-uniform sampling. Thus, it is quite necessary to apply non-uniform BTT sampling to address the drawbacks of uniform sampling. 


\section{Mathematical Model of the Non-Uniform BTT Sampling}

\subsection{Representation of Blade Tip Displacements}

As shown in Figure 1, BTT samples are acquired from $I$ probes mounted circumferentially around a rotor with $K$ blades. An additional probe $r$ is mounted in front of the shaft as a reference sensor. There is a white marker line milled on the shaft, so that the reference sensor can measure the once-per-revolution signal. The relative angular position in the casing between probe 0 and probe $i(0 \leq i<I)$ is denoted as $\alpha_{i}$. Without loss of generality, the angular position of probe 0 is set to 0 . Similarly, the location of blade $k$ is set as $\theta_{k}$. Since blades are assumed to be equally spaced, one will have $\theta_{k}=\theta_{0}+2 \pi k / K, k \in\{0,1, \ldots, K-1\}$, where generally $\theta_{0}=0$.

The concept of the BTT method is to measure the arrival time of the tip of a vibrating blade as it passes a probe. The expected blade arrival times for a single non-vibrating blade at any probe are determined by the rotation speed, blade tip radius and angular position of the probe. When there are vibrations, any deviations from these expected arrival times indicate blade vibrations with respect to the hub. These deviations are recorded by each probe to calculate a time-series of blade tip displacements and further used to analyze blade vibration characteristics.

Assuming that $t_{i, n}^{k}$ represents the actual arrival time when the blade $k$ passes in front of the probe $i$ at the $n$-th rotation, and the expected arrival time for a non-vibrating blade is denoted as $\bar{t}_{i, n}^{k}$. When the rotating speed $f_{r}$ is constant, the expected arrival time can be formulated as follows [18]:

$$
\bar{t}_{i, n}^{k}=\frac{1}{2 \pi f_{r}}\left(\alpha_{i}+2 \pi n-\theta_{k}\right)
$$

There is an angular deflection $d\left(t_{i, n}^{k}\right)$ at time $t_{i, n}^{k}$ due to the blade vibration. Thus one will obtain:

$$
t_{i, n}^{k}=\frac{1}{2 \pi f_{r}}\left(\alpha_{i}+2 \pi n-\theta_{k}-d\left(t_{i, n}^{k}\right)\right)
$$

Subtracting Equation (2) from Equation (1), one will have:

$$
d\left(t_{i, n}^{k}\right)=2 \pi f_{r}\left(\bar{t}_{i, n}^{k}-t_{i, n}^{k}\right)=2 \pi f_{r} \Delta t_{i, n}^{k}
$$

As shown by Equation (2), the sampling time $t_{i, n}^{k}$ depends on the signal itself. Using $d\left(\bar{t}_{i, n}^{k}\right)$ instead of $d\left(t_{i, n}^{k}\right)$ is a feasible way to reduce the complexity of following signal processes.

\subsection{Mathematical Model of the Non-Uniform BTT Sampling}

Assuming that a real continued vibration signal of blade $k$ is denoted as $r_{k}(t)$. Since the following mathematical model is true for any blade $k$, an arbitrary blade can be considered. Thus, to simplify notations, the index $k$ is dropped in the following sections. Based on Equation (3), the blade vibration signal $r(t)$ will be sampled at the time $\bar{t}_{i, n}$. If the rotation speed $f_{r}$ is constant, the non-uniform BTT sampling function can be formulated as follows:

$$
x(t)=r(t) \sum_{i=1}^{I-1} \sum_{n=0}^{N-1} \delta\left(t-\bar{t}_{i, n}\right)=r(t) \sum_{i=1}^{I-1} \sum_{n=0}^{N-1} \delta\left(t-\frac{n}{f_{r}}-\frac{\alpha_{i}}{2 \pi f_{r}}\right)
$$


where $\delta(\cdot)$ is the Dirac delta function. $x(t)$ denotes the blade tip displacements, i.e., $x\left(t_{i, n}\right)=d\left(t_{i, n}\right) R$, where $R$ is the distance between the blade tip and the rotating center. Equation (4) is a sum of $I$ uniform sample streams. Here a stream associates with a time-series of blade tip displacements recorded by a probe. $a_{i} / 2 \pi f_{r}$ represents the delay of the $i$-th sample stream. This delay depends strongly on the angular position of probe $i$ and the rotation speed. In particular, $a_{0} / 2 \pi f_{r}=0$.

In practice, the blade vibration is often dominated by a single frequency. The blade response can then be modeled as a single degree of freedom formulation:

$$
r(t)=A e^{j\left(2 \pi f_{0} t+\varphi\right)}
$$

where $f_{0}$ is the dominant frequency. However, the real frequency spectrum of such a blade vibration may have a narrow bandwidth $B_{0}$. As shown in Figure 3a, a frequency window with a bandwidth $B$ and a central frequency $f_{c}$ is selected to represent the blade vibration signal, where $f_{c}$ is an estimated vibration frequency. The guard-band $B_{g}=B-B_{0}$ is set to prevent aliasing. The lowest and highest positive frequencies of the window are defined as $f_{L}=f_{c}-B / 2$ and $f_{H}=f_{c}+B / 2$, respectively. Therefore the blade tip-timing sampling becomes a band-pass sampling.

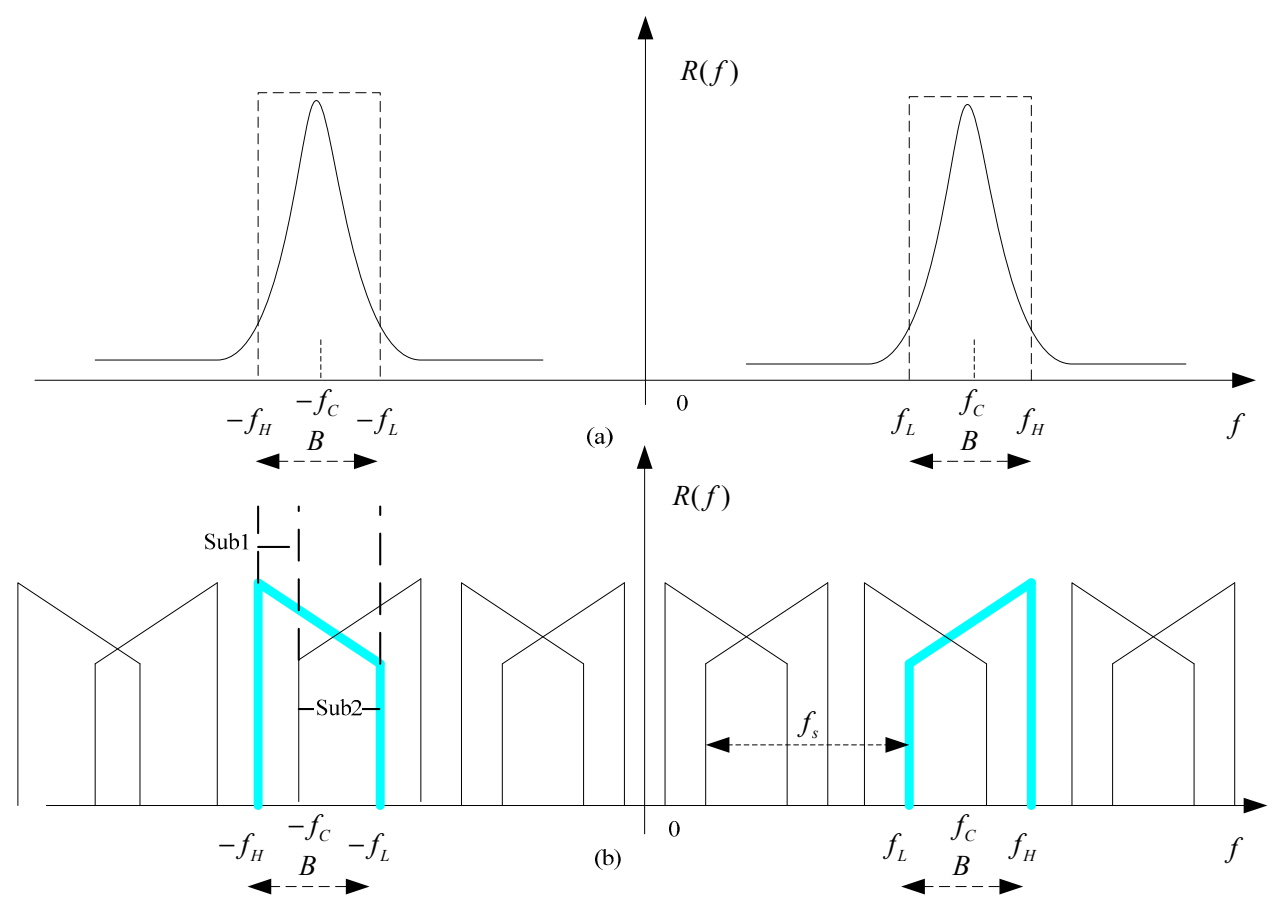

Figure 3. (a) The original blade vibration signal; (b) The spectrum of one sample stream of the non-uniform sampling.

\section{Reconstruction of the Periodically Non-Uniform Band-Pass Sampling}

\subsection{Mathematical Model of the Reconstruction}

Reference [21] indicated that to reconstruct any sampled signal in the time domain it is necessary to apply an interpolating function to the sampled signal. The non-uniform sampling comprises $I$ uniform 
sample streams. For each stream, an interpolating function is required, as shown in Figure 4. Thus the reconstructed signal can be defined as follows:

$$
r_{1}(t)=\sum_{i=1}^{I-1} \sum_{n=0}^{N-1} r\left(\frac{n}{f_{r}}+\frac{\alpha_{i}}{2 \pi f_{r}}\right) S_{i}\left(t-\frac{n}{f_{r}}-\frac{\alpha_{i}}{2 \pi f_{r}}\right)
$$

where $S_{i}(\cdot)$ is the interpolating function, $r_{1}(t)$ represents the reconstructed signal.

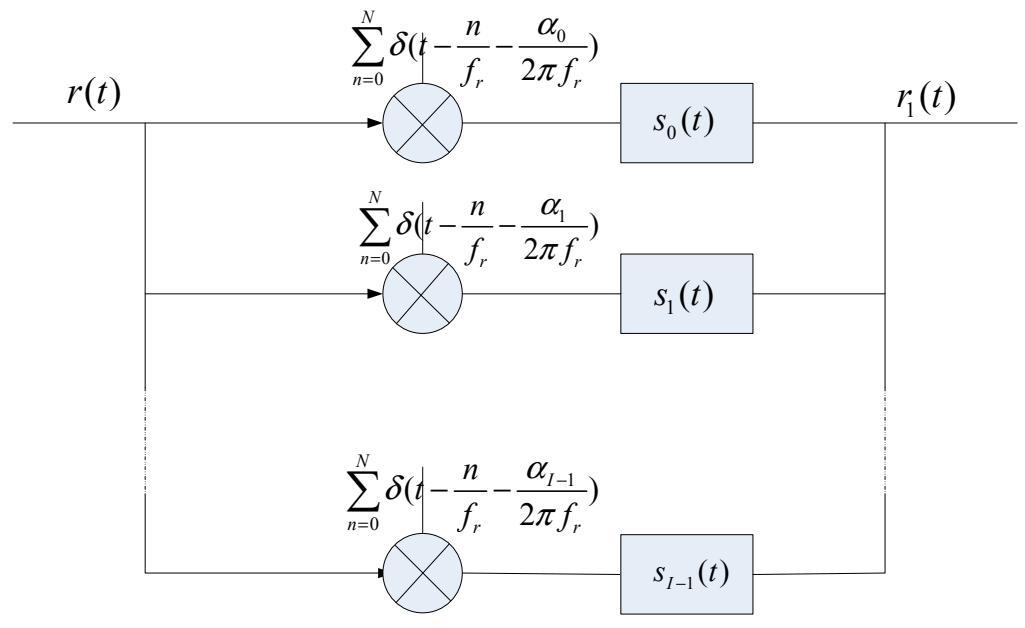

Figure 4. Reconstruction of the periodically non-uniform sampling.

Since it is difficult to directly derive analytical solutions of interpolating functions in the time-domain, one could solve this problem in the frequency-domain. The Fourier transform of $r_{1}(t)$ can be formulated as follows:

$$
R_{1}(f)=\sum_{i=0}^{I-1} f_{r} S_{i}(f) \sum_{n=-\infty}^{\infty} R\left(f-n f_{r}\right) e^{-j n \alpha_{i}}
$$

The frequency spectrum of one sample stream is shown in Figure 3b. The original signal is marked by the blue color. It is easily observed that uniform sampling leads to a periodical replication of the original signal, and a finite number of replicas are intersected in ranges of the original signal, i.e., $f_{L}<f<f_{\mathrm{H}}$ and $-f_{H}<f<-f_{\mathrm{L}}$. It must be noted that the aliased contribution is the same for each sample stream except for a different phase shift. To reconstruct the original signal, all unwanted replicas in these ranges must be removed such that $R_{1}(f)=R(f)$.

To achieve that, the ranges of the original signal are separated into a number of sub-bands. Each sub-band has different intersected parts of replicas, which strongly depend on $B / f_{r}$ [22]. In each sub-band, simultaneous equations are built to ensure that all unwanted replicas sum to zero upon summation of all post-interpolation sample stream. It is assumed that the range of the $j$ th sub-band with $N_{j}$ interacted replicas is denoted as $f_{L}^{j}<f<f_{H}^{j}$. Based on Equation (7), simultaneous equations in this sub-band are built:

$$
\left\{\begin{array}{c}
\sum_{i=0}^{I-1} f_{r} S_{i, j}(f)=1 \\
\sum_{i=0}^{I-1} S_{i, j}(f) e^{-j n \alpha_{i}}=0, \forall n \in A, n \neq 0
\end{array}\right.
$$


where $S_{i, j}(f)$ represents the $j$ th element of $S_{i}(f)$ in the corresponding sub-band. The first equation indicates that the original blade vibration signal in this sub-band should be remained. Other $N_{j}-1$ equations are built to remove unwanted replicas, where $A$ denotes the index set of unwanted replicas, i.e., $A=\left\{n \mid\left(f_{H}+n f_{r} \geq f_{H}^{j} \wedge f_{L}+n f_{r} \leq f_{L}^{j}\right) \vee\left(-f_{L}+n f_{r} \geq f_{H}^{j} \wedge-f_{H}+n f_{r} \leq f_{L}^{j}\right)\right\}$.

\subsection{Blade Vibration Reconstruction by Using Two Probes}

Reference [22] has derived a generalization of the analytical solution of $S_{i, j}(t)$. However, it is relatively complicated when $B>f_{r}$. The calculations of partitioning sub-bands and solving equations are rapidly increasing with the increases of $B / f_{r}$. It also requires more sample streams to obtain a solution of Equation (8). This requirement conflicts with the limited number of probes in BTT. In order to solve this problem, this paper will propose a non-uniform BTT sampling with only two probes. It is totally enough to reconstruct a blade vibration signal in practice.

In Equation (8), if the number of replicas is greater than the number of probes, i.e., $N_{j}>I=2$, there will be no solutions for each $S_{i, j}(f)$ since alias-free interpolation requires the solution of more than $I$ equations using only $I$ variables. In contrary, there will at least one solution for $S_{i, j}(f)$ if $N_{j} \leq I=2$. Instantaneously, reference [22] demonstrated that $N_{j}$ increases with the increases of $B / f_{r}$, i.e., $N_{j} \propto B / f_{r}$. Especially, when $B / f_{r}=1$, one will have $N_{j}=2$. Hence, if $B=f_{r}>B_{0}$, that is, if the rotation speed is more than the narrow bandwidth of the blade vibration signal, it is enough to use two probes to reconstruct a blade vibration signal non-aliasing.

Generally, three or four probes are selected in practice. In the view of this paper, only the case $B=f_{r}<B_{0}$ needs more probes simultaneously to reconstruct a signal. Otherwise the blade vibration signal can be reconstructed by using two arbitrary probes. The corresponding reconstruction formula has derived based on the results in [23]:

$$
r_{1}(t)=\sum_{n=-\infty}^{\infty} r\left(\frac{n}{B}\right) S\left(t-\frac{n}{B}\right)+r\left(\frac{n}{B}+\frac{\alpha_{i}}{2 \pi B}\right) S\left(-t+\frac{n}{B}+\frac{\alpha_{i}}{2 \pi B}\right)
$$

where $S$ is the interpolation as follows:

$$
\begin{aligned}
& S(t)=\frac{\cos \left(2 \pi\left(m B-f_{L}\right) t-m \alpha_{i} / 2\right)-\cos \left(2 \pi f_{L} t-m \alpha_{i} / 2\right)}{2 \pi B t \operatorname{Sin}\left(m \alpha_{i} / 2\right)} \\
& +\frac{\cos \left(2 \pi\left(B+f_{L}\right) t-(m+1) \alpha_{i} / 2\right)-\cos \left(2 \pi\left(m B-f_{L}\right) t-(m+1) m \alpha_{i} / 2\right)}{2 \pi B t \operatorname{Sin}\left((m+1) m \alpha_{i} / 2\right)}
\end{aligned}
$$

Here $m=\left\lceil 2 f_{L} / B\right\rceil$. The ceiling operator $\lceil X\rceil$ denotes the smallest integer not less than $X$. In Equation (10), important constraints should be noticed, i.e., $\sin \left(m \alpha_{i} / 2\right) \neq 0$ and $\sin \left((m+1) \alpha_{i} / 2\right) \neq 0$. Otherwise the interpolation will be meaningless due to the dividing zero. This way, the angular positions of probes should be selected carefully to avoid incorrect reconstructions. In addition, it must be noted that the central frequency $f_{c}$ and the bandwidth $B$ (equal to the rotation speed) of the frequency window should be known a priori. Generally, the central frequency $f_{c}$ can be estimated by FEM methods or modal frequency identification algorithms. 


\section{Numerical Simulations}

The reconstruction performances of non-uniformly under-sampled BTT data will be evaluated in terms of the sampling frequency, the blade vibration frequency, the blade vibration bandwidth, the probe static offset and the number of samples. Since the blade vibration is a narrowband signal, in order to provide more insight, the vibration signal in these simulations is simply replaced by a typical band-pass signal, i.e., $f(t)=\sin c\left(B_{0} t\right) \sin \left(2 \pi f_{c} t\right)$ [16]. All conclusions are also established for real blade vibrations. With the setting of $B_{0}=50 \mathrm{~Hz}, f_{c}=827 \mathrm{~Hz}$, Figure 5 shows the frequency spectrum of this original signal. The related simulation parameters of BTT are shown in Table 1. In this case, the individual sampling rate will be equal to the rotating speed, i.e., $f_{r}=5000 \mathrm{n} / \mathrm{min}=83.3 \mathrm{~Hz}$.

The aliasing of under-sampling is evaluated first. The frequency spectra of all three uniform sample streams with $f_{r}=83.3 \mathrm{~Hz}$ are shown in Figure 6. The curves of various probes are the superposition of two replicas of the original signal with different phase shift, which are significantly different due to aliasing.

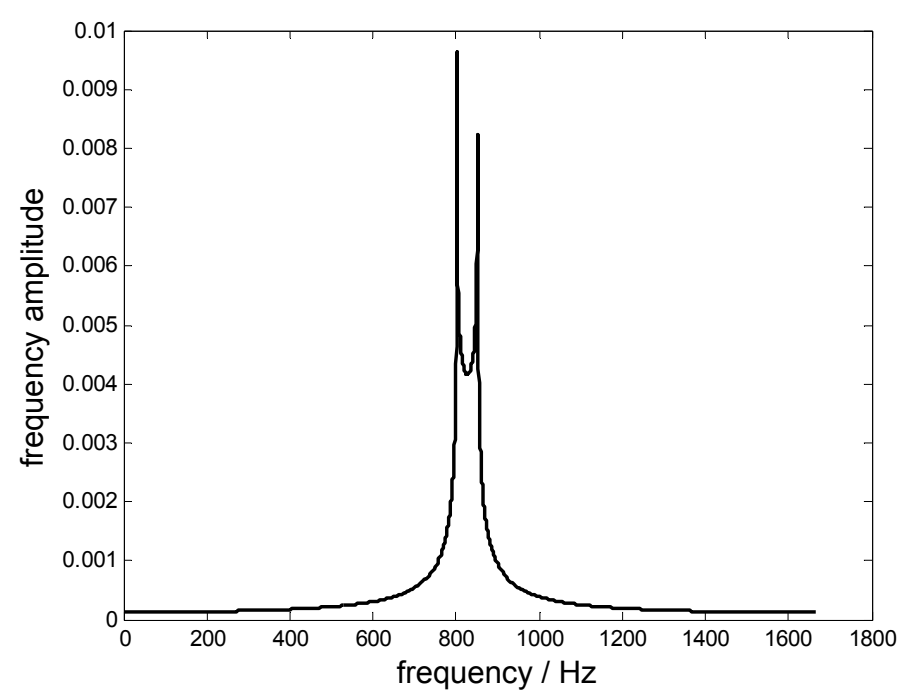

Figure 5. The frequency spectrum of the original signal.

Table 1. Experimental Setting.

\begin{tabular}{cc}
\hline Parameters & Properties \\
\hline Material of the bladed-disk & Type 45 steel \\
The number of blades & 16 \\
The length of each blade & $45 \mathrm{~mm}$ \\
The width of each blade & $20 \mathrm{~mm}$ \\
The thickness of each blade & $2 \mathrm{~mm}$ \\
The distance of the blade tip to the center & $95 \mathrm{~mm}$ \\
The rotating speed & $5000 \mathrm{rpm}$ \\
The angular position of probe 1 & $0^{\circ}$ \\
The angular position of probe 2 & $120^{\circ}$ \\
The angular position of probe 3 & $240^{\circ}$ \\
\hline
\end{tabular}




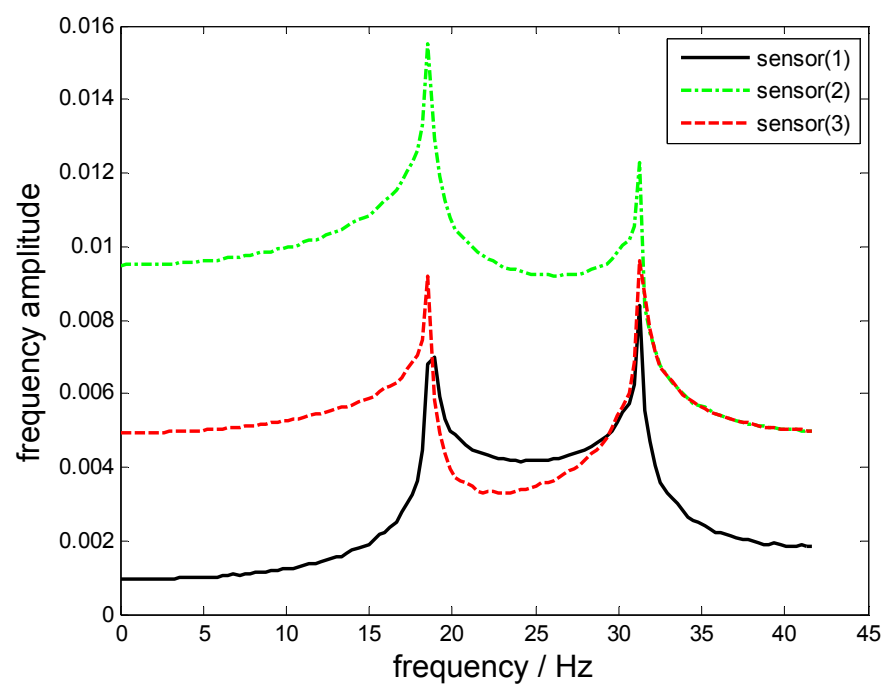

Figure 6. The frequency spectra of sample streams with $f_{r}=83.3 \mathrm{~Hz}$.

\subsection{Definition of the Reconstruction Error}

In this section, no-uniformly under-sampled BTT signals from Probes $(1,2)$ are used to reconstruct the original signal. Uniform BTT signals from probes $(1,2,3)$ are also used to reconstruct the original signal based on the Shannon sampling theorem [14,23]. Compared results between reconstructed signals and the original signal are shown in Figure 7. The corresponding local logarithmic reconstruction error [14] is also shown in Figure 7, defined by:

$$
e(x)=\log \left(\left|r(t)-r_{1}(t)\right|\right)
$$

It can be easily found that the reconstructed signals by Probes $(1,2)$ are proximate to the original signal. The corresponding local logarithmic reconstruction error is relatively small and uniform in comparison with that of the uniform reconstruction. Since interpolations require many terms of samples to be evaluated, using a finite number of samples will affect the accuracy of the reconstructed signal, especially the local signals close to the start and end of samples. The uniform reconstruction suffers more loss since the kernel function $\sin c(\bullet)$ used by the Shannon sampling theorem is known to decay very slowly. However, a promising way out is to apply a time-limited reconstruction kernel function $[13,16]$ to replace the $\sin c(\bullet)$ function so that only few samples have to be involved.
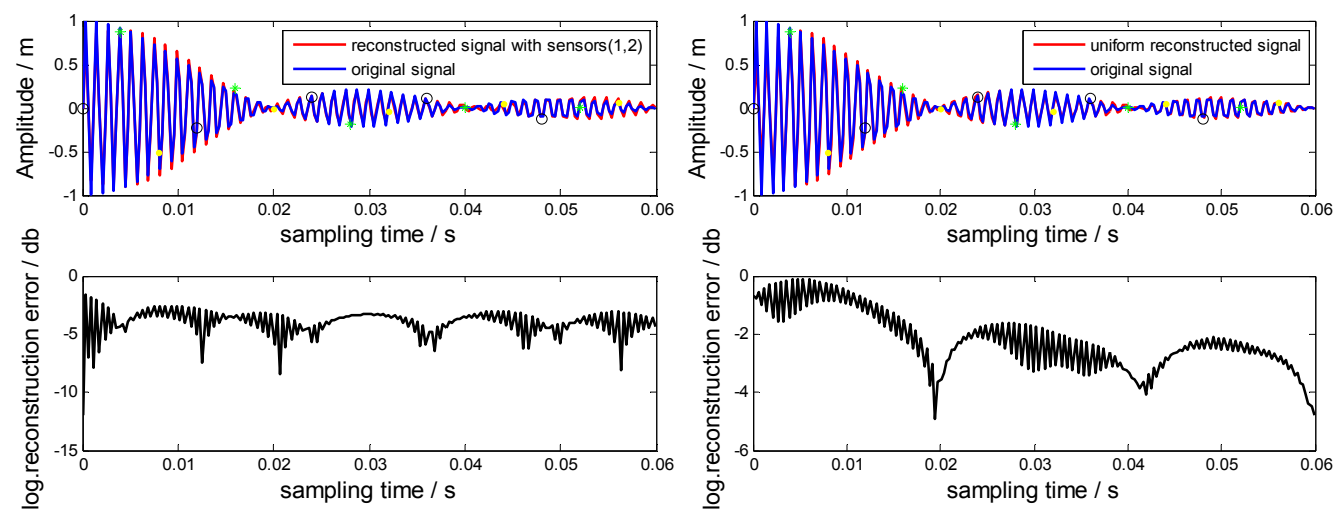

Figure 7. Reconstructed signals and local logarithmic reconstruction errors. 
Figure 8 shows frequency spectra of reconstructed signals and the original signal. The non-uniform reconstructed signal is almost as the same with the original signal. However, a mutation still occurs in the boundaries of the selected frequency window.

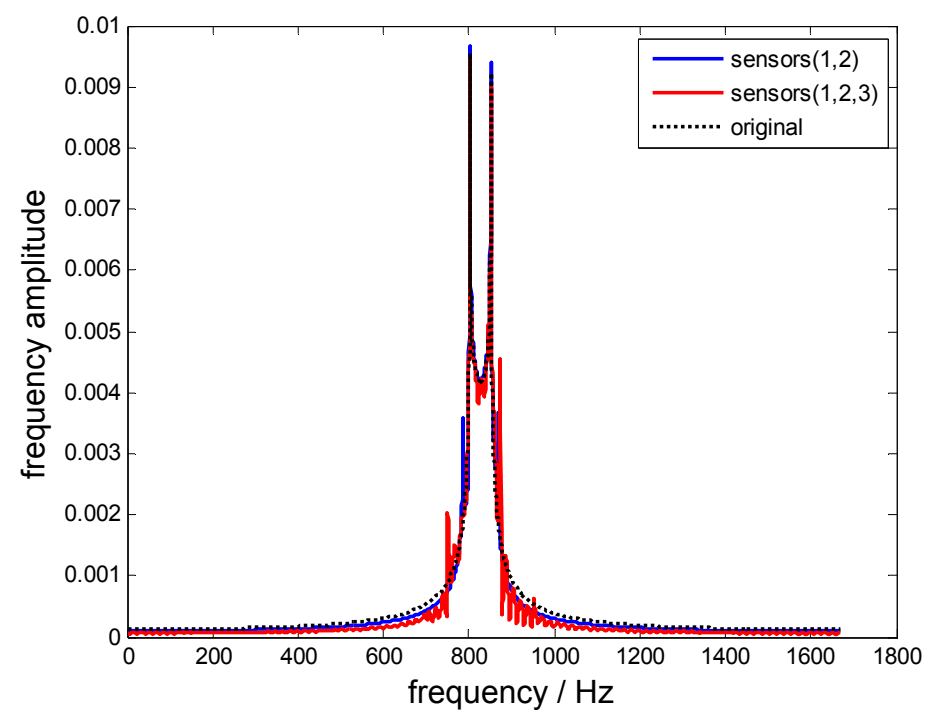

Figure 8. Frequency spectra of reconstructed signals.

\subsection{Reconstruction Error Affected by the Bandwidth $B_{0}$}

In this section, simulations are done to estimate the reconstruction error affected by the bandwidth $B_{0}$. In Equation (9), the bandwidth $B$ of the frequency window depends on the sampling rate $f_{r}$, i.e., $B=f_{r}=83.3 \mathrm{~Hz}$. Setting $B_{0}=100 \mathrm{~Hz}>B$, the corresponding reconstructed signals in the time-domain and frequency-domain are shown in Figures 9 and 10, respectively.
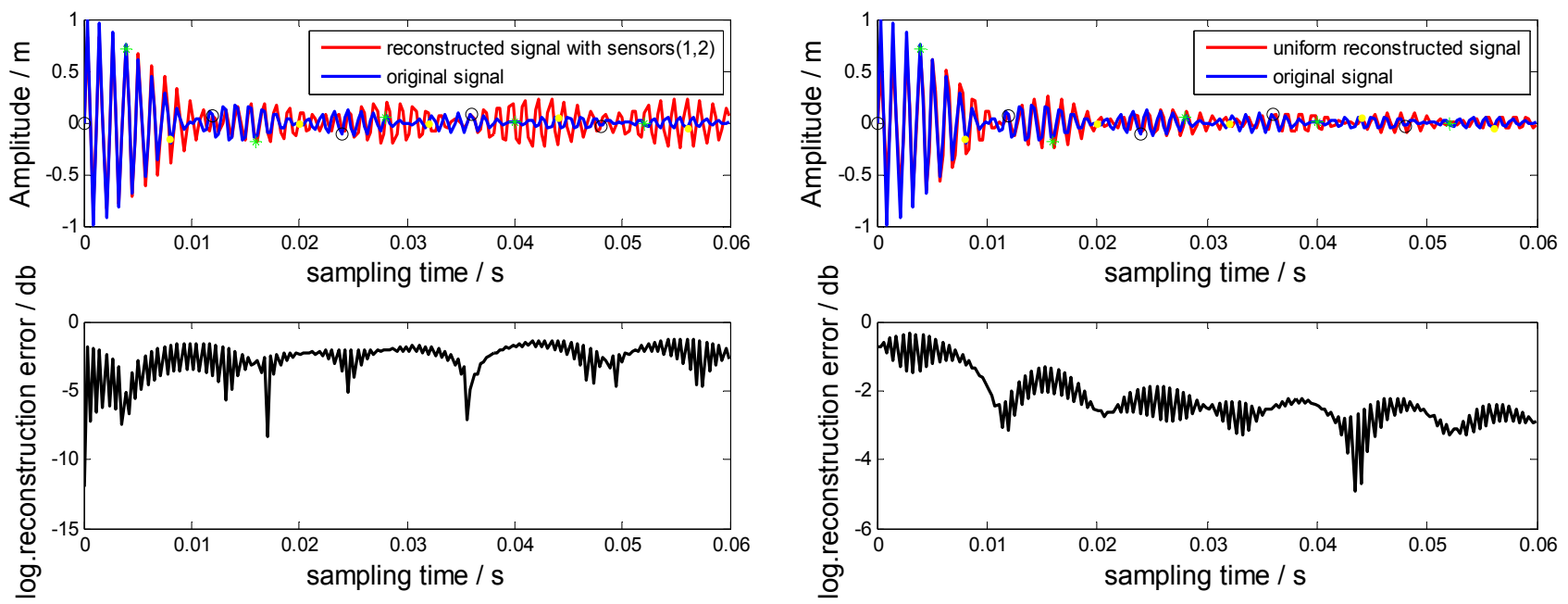

(a)

(b)

Figure 9. Reconstructed signals and the original signal with $B_{0}=100 \mathrm{~Hz}$ for (a) the non-uniform sampling and (b) the uniform sampling. 


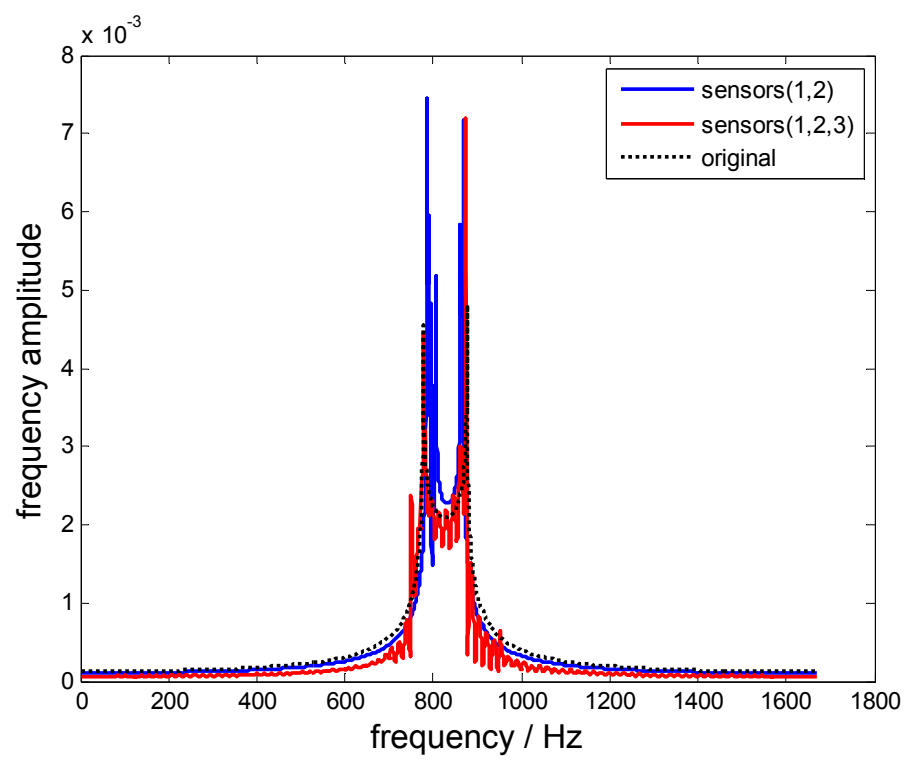

Figure 10. Frequency spectra of reconstructed signals and the original signal with $B_{0}=100 \mathrm{~Hz}$.

Obviously, there are significant differences between the original signal and the non-uniform reconstructed signal. The reconstruction is aliasing. However, the reconstruction error of the uniform reconstructed signal is smaller than that of the non-uniform one. This is because the uniform sampling using three probes has a wider bandwidth $B_{1}$ of the frequency window, where $B_{1}>B$. In order to obtain a non-aliasing reconstructed signal for this case, there are two feasible ways. First, one can increase the rotating speed to increase the bandwidth $B$. Second, if increasing the rotation speed is not allowed since it will change the blade vibration characteristics, probes could be added to ensure there are solutions for $S_{i, j}(f)$ in Equation (8) when $B>f_{r}$.

\subsection{Reconstruction Error Affected by the Vibration Frequency $f_{0}$}

The blade vibration frequency $f_{0}$ is an important parameter reflecting the health of a blade. It is often estimated by various methods $[7,8,11]$. However, the uncertainties between estimated value $\bar{f}_{0}$ and the original one $f_{0}$ may cause a severe reconstruction error. The blade vibration frequency is set as $f_{0}=827 \mathrm{~Hz}$. The estimated frequency is assumed as $\bar{f}_{0}=800 \mathrm{~Hz}$, so that the central frequency is selected as $f_{c}=\bar{f}_{0}=800 \mathrm{~Hz}$. The uniform and non-uniform reconstructed signals are shown in Figure 11. The non-uniform reconstruction error is relatively large, since the frequency spectrum of this signal has shifted from the original position as shown in Figure 12. It has become a superposition of a replica and the original signal. In contrast, the uniform reconstruction has little changes in comparison with that shown in Figure $7 \mathrm{~b}$. In this case, the bandwidth $B_{1}$ of the frequency window of the uniform reconstruction is wide enough, such that the original signal still locates at the window range $\left[\bar{f}_{c}-B_{1} / 2, \bar{f}_{c}+B_{1} / 2\right]$ as shown in Figure 12 . For non-uniform sampling, an adjustment of the estimated frequency should be proposed to reduce the reconstruction error, which will be the next work. 

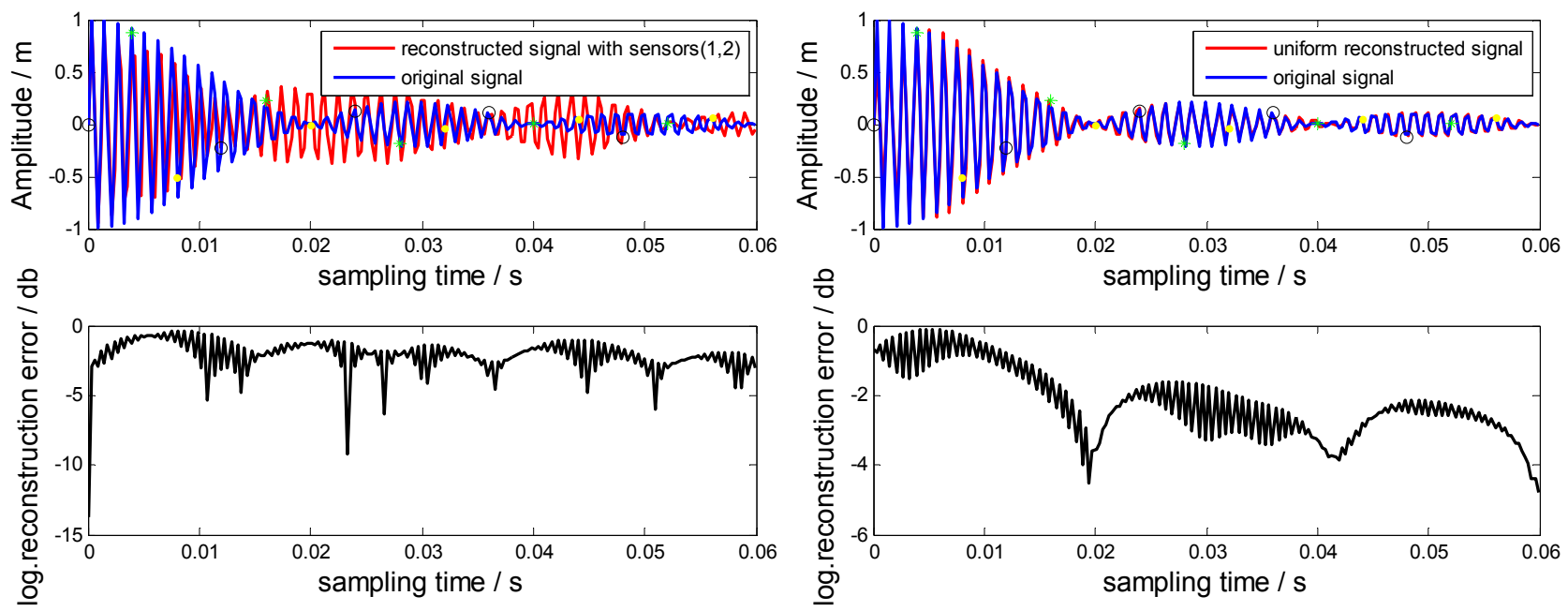

Figure 11. Reconstructed signals and the original signal with $\bar{f}_{c}=800 \mathrm{~Hz}$.

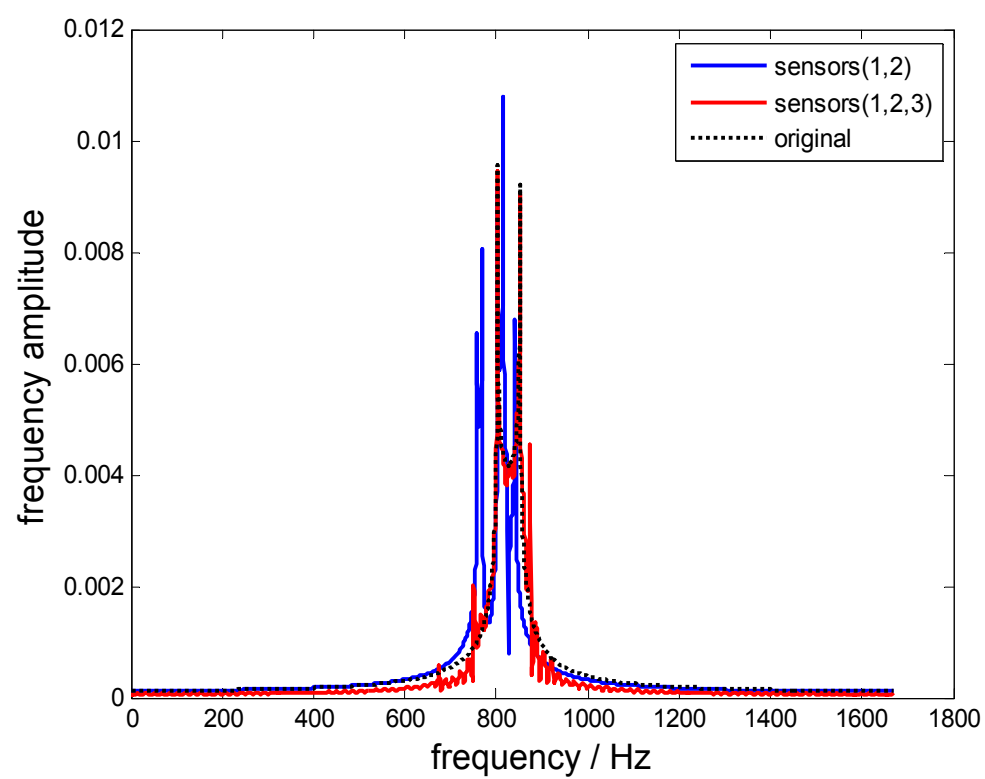

Figure 12. Frequency spectra of reconstructed signals with $\bar{f}_{c}=800 \mathrm{~Hz}$.

\subsection{Reconstruction Error Affected by the Number of Samples}

In practice, a reconstruction error will occur since only a finite number of samples are used to reconstruct a signal based on Equation (9). In order to reduce this error, frequency spectra of reconstructed signals with various numbers of samples are investigated in Figure 13. To identify different curves, each curve is shifted by $100 \mathrm{~Hz}$ from each other. It is easily found that with the increases of the number of samples, the reconstructed signal is more approximate to the original signal. Generally, 200 samples of each uniform sample stream are enough to reconstruct the original signal approximately. 


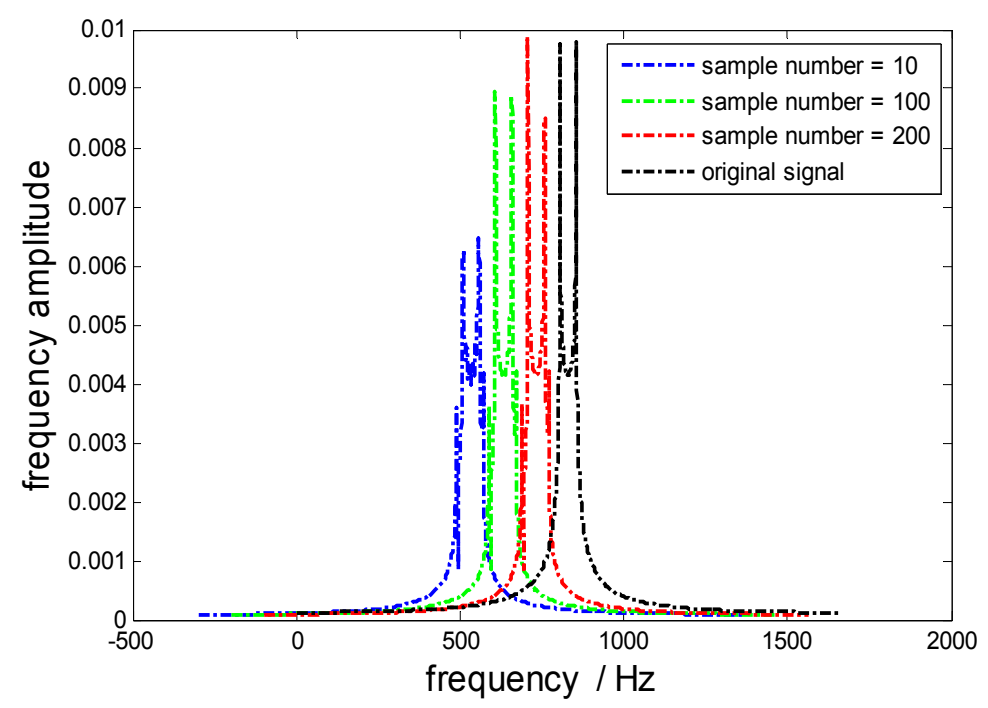

Figure 13. Frequency spectra of reconstructed signals with various numbers of samples.

\subsection{Reconstruction Error Affected by the Probe Static Offset}

The accurate reconstruction also depends on the accuracy of angular positions of probes. Setting the probe static offset $\Delta \alpha_{i}=2^{\circ}$, frequency spectra of reconstructed signals are shown in Figure 14. The significant reconstruction error indicates the probe static offset due to manufacturing tolerances should be kept minor.

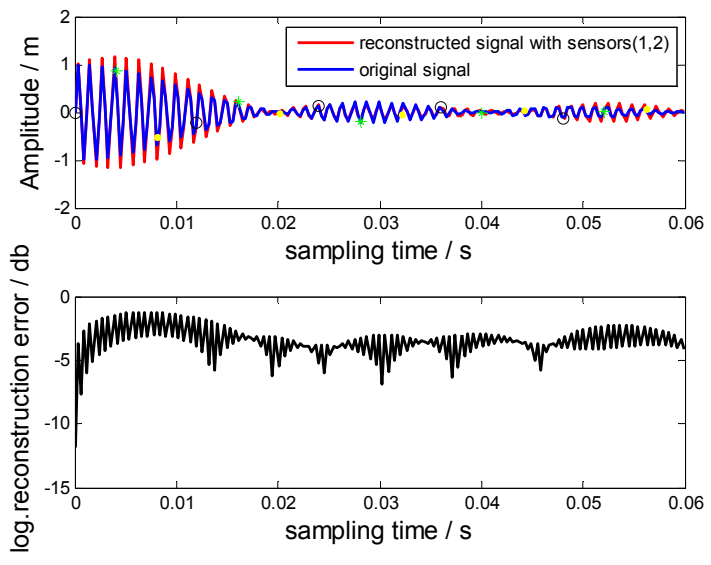

(a)

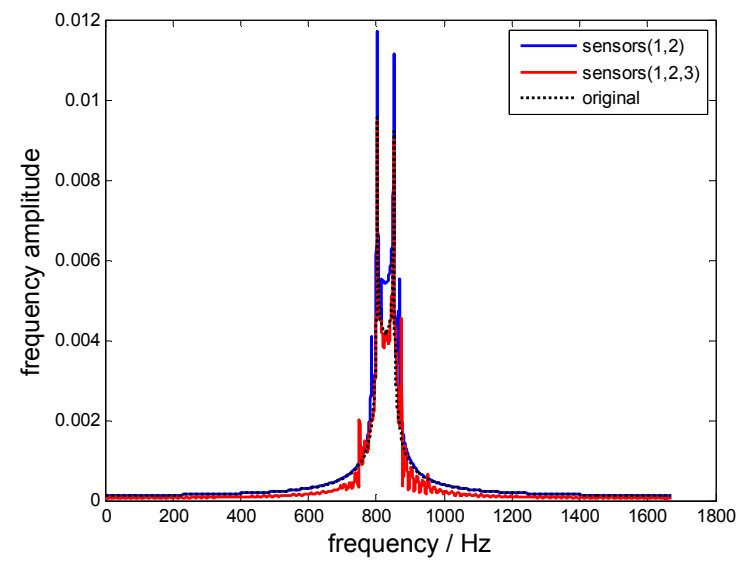

(b)

Figure 14. Reconstructed signals with the probes offset in (a) time-domain and (b) frequency-domain.

\subsection{Summary}

In this section, the aliasing of under-sampling has been evaluated. Then the reconstruction performances are evaluated in terms of the blade vibration frequency, the blade vibration bandwidth, the probe static offset and the number of samples. The results show that the proposed method is feasible for reconstructing original signals by using more than 200 samples of each probe. Additionally, assuring that the original blade vibration frequency $f_{0}$ locates within the specified 
frequency window $\left[f_{L}, f_{H}\right]$ is very important to decrease reconstruction errors, and probe static offsets should be kept small too.

\section{Experiments}

As shown in Figure 15, an experimental set-up is built to validate the feasibility of the proposed method. In order to compare with the uniform sampling, three optical-fiber probes are embedded equally in the circular bracket to sample arrival times. Using two arbitrary probes forms a non-uniform sampling. The additional optical-fiber probe is placed close to the rotating shaft for sampling reference time.

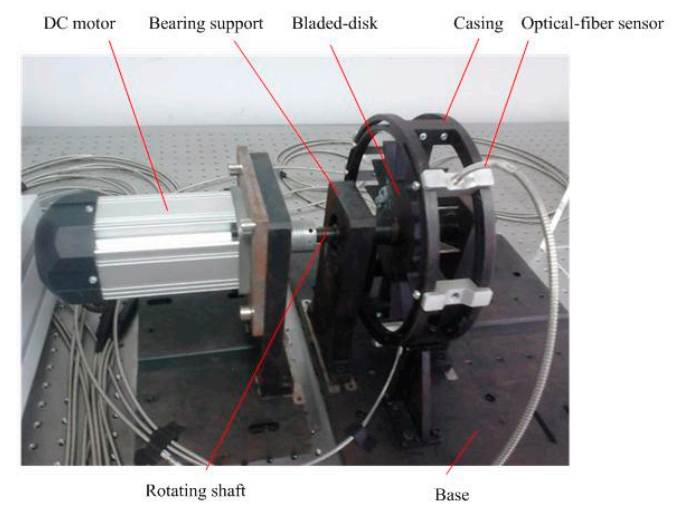

Figure 15. The test rig.

Other detailed experimental parameters are shown in Table 1. The blade vibration frequency is estimated by FEM simulations, i.e., $\bar{f}_{0}=827 \mathrm{~Hz}$, such that the central frequency is selected as $f_{c}=\bar{f}_{0}$. A long time test is done to collect at least 3000 samples. Four-channel time impulse signals are collected and all-blade tip displacements are calculated based on Equation (3). Here vibration signals of Blade 0 are considered. The frequency spectra of all three uniform sample streams are shown in Figure 16. The peaks of the spectra are located at $\bar{f}=0$. Based on the sampling theorem, the blade vibration frequency can be inferred as $f_{0}=n f_{r}+\bar{f}=n f_{r}$.

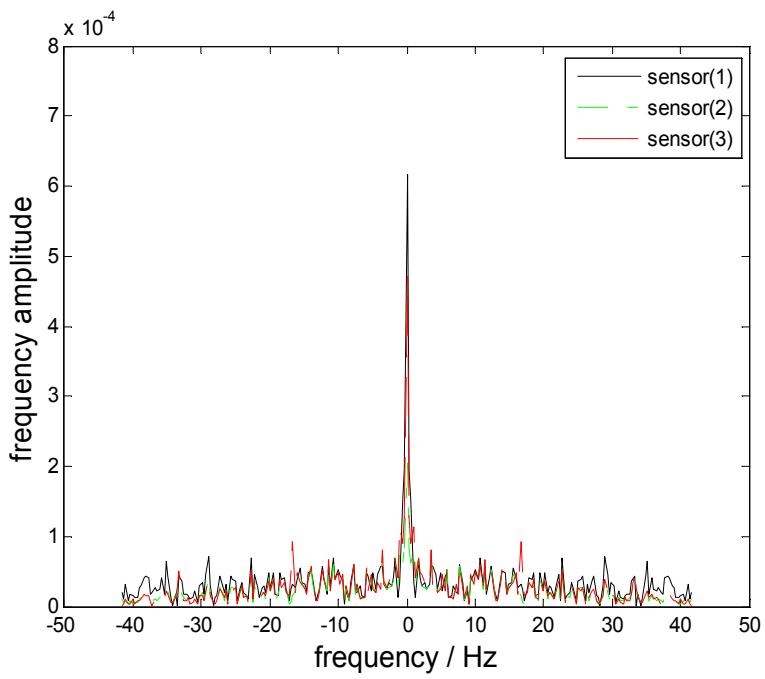

Figure 16. The frequency spectra of sample streams. 
The reconstructed signals from various probes are shown in Figure 17. The reconstructed signal curves using probes $(1,2)$ and probes $(1,3)$ are similar. However, the curve using probes $(2,3)$ is not so precise. The measurement noise and probe static offsets forced on these probes could induce significant reconstruction errors. These errors have been also investigated in [21,24]. The results in these papers could be further used to optimize the reconstruction process. We will also focus on decreasing these harmful impacts in our future work.
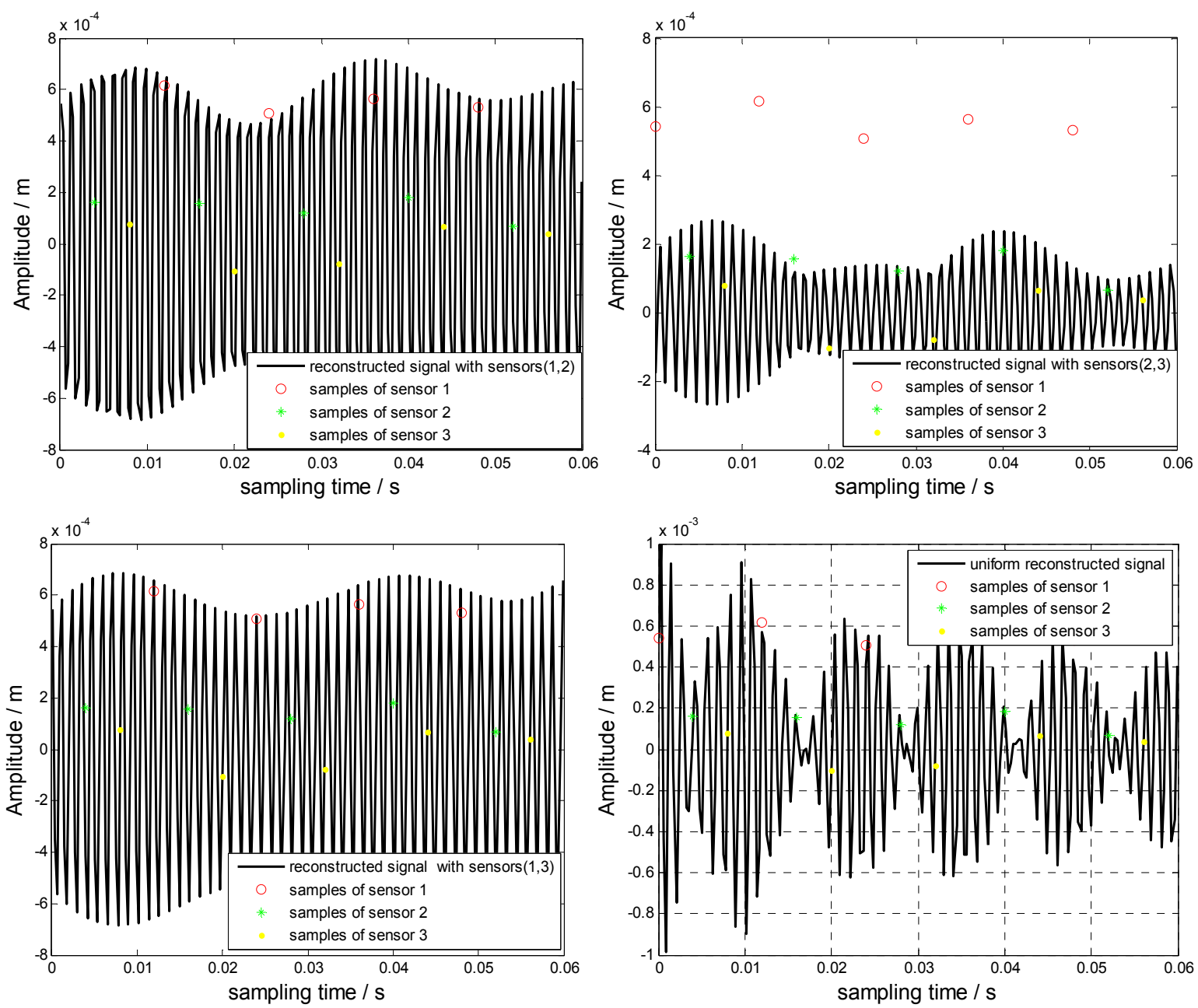

Figure 17. Reconstructed Signals.

Additionally, the uniform reconstructed signal is also different from the non-uniform one in the time domain as shown in Figure 17. Nevertheless, the frequency spectra in Figure 18 show that the blade vibration frequency $f_{0}=833.5 \mathrm{~Hz}$ can be precisely detected from these two different reconstructed signals, which validates the feasibility of the proposed method. However, it must be noted that different notable frequencies exist nearby $833.5 \mathrm{~Hz}$, which causes reconstructed signals to be distorted. These differences are dominated by the measurement noise propagated in these two different approaches, therefore, to improve the signal-to-noise ratio and optimizing the reconstruction process is very important, which is one key point of our future work. 


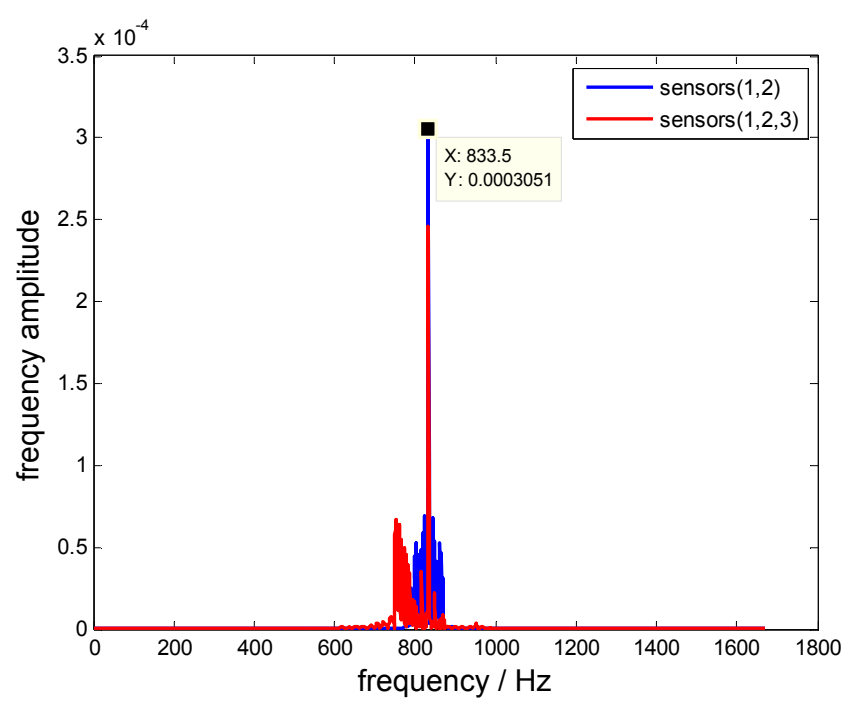

Figure 18. Frequency spectra of reconstructed signals.

\section{Conclusions}

The blade tip-timing method has become an important non-contact way to monitor blade vibrations online. In order to obtain accurate vibration characteristics, two key points are important. First, it is well known that under-sampling is an intrinsic drawback of BTT methods. Thus a signal reconstruction based on BTT data is required to solve this bottleneck problem. Second, non-equally mounted probes have been used to measure synchronous and asynchronous vibrations instantaneously. However, nowadays little work has been done on reconstructing non-uniformly under-sampled BTT signals. In this paper, a novel reconstruction of non-uniformly under-sampled BTT data is proposed based on the periodically non-uniform sampling theorem. Firstly, all-blade vibration displacements using optical-fiber probes are calculated based on BTT methods. Then a mathematical model of a non-uniform BTT sampling process is built. It can be treated as the sum of certain uniform sample streams. For each stream, an interpolating function is required to prevent aliasing in the reconstructed signal. To achieve that, sub-bands are defined in the range of the original signal. Each sub-band has different intersected parts of replicas of the original signal. Next, it builds simultaneous equations of all interpolating functions in each sub-band and ultimately derives the solutions to remove unwanted replicas of the original signal caused by the sampling. In the end, numerical simulations and experiments are done to validate the feasibility of the proposed method. The main results are summarized as follows:

1. A non-aliasing reconstruction of non-uniformly under-sampled BTT data based on the periodically non-uniform sampling theorem is presented.

2. The accuracy of the reconstructed vibration signal depends on the sampling frequency (the rotation speed), the blade vibration frequency, the blade vibration bandwidth, the probe static offset and the number of samples.

3. In practice, a blade vibration signal can be particularly reconstructed by non-uniformly undersampled BTT data acquired from only two probes if the blade vibration frequency of the blade is known in advance. 


\section{Acknowledgments}

This study was co-supported by the National Basic Research Program of China (Grant No. 2015CB057400, the Open Foundation of Hunan Province Key Laboratory of Health Maintenance of Mechanical Equipment (Grant No. 201201) and the State Key Laboratory of Robotics.

\section{Author Contributions}

This work has been done in collaboration between all authors. Zheng $\mathrm{Hu}$, Jun Lin and Zhong-Sheng Chen designed the proposed method and prepared the manuscript. Yong-Min Yang supervised the research. Xue-Jun Li interpreted and analyzed the corresponding results. Both authors prepared, revised and approved the final submission.

\section{Conflicts of Interest}

The authors declare no conflict of interest.

\section{References}

1. Grant, K.R.; Ivey, P. The experimental validation of tip-timing techniques for condition monitoring of HCF in gas turbine aero-engines. In Proceedings of the 38th AIAA/ASME/SAE/ASEE Joint Propulsion Conference \& Exhibit, Indianapolis, IN, USA, 7-10 July 2002.

2. Knappett, D.; Garcia, J. Blade tip timing and strain gauge correlation on compressor blades. Proc. Inst. Mech. Eng. Part G J. Aerosp. Eng. 2008, 222, 497-506.

3. Lawson, C.; Ivey, P. Tubomachinery blade vibration amplitude measurement through tip timing with capacitance tip clearance probes. Sens. Actuators A Phys. 2005, 118, 14-24.

4. García, I.; Beloki, J.; Zubia, J. An Optical Fiber Bundle Sensor for Tip Clearance and Tip Timing Measurements in a Turbine Rig. Sensors 2013, 13, 7385-7398.

5. Zielinski, M.; Ziller, G. Noncontact Blade Vibration Measurement System for Aero Engine Application. Available online: http://www.mtu.de/en/technologies/engineering_news/ development/Ziller_Noncontact_blade_vibration_en.pdf (accessed on 20 January 2014).

6. Cardwell, D.N.; Chana, K.S.; Russhard, P. The Use of Eddy Current Sensors for the Measurement of Rotor Blade Tip Timing: Sensor Development and Engine Testing. In Proceedings of the ASME Turbo Expo 2008: Power for Land, Sea, and Air. American Society of Mechanical Engineers, Berlin, Germany, 9-13 June 2008.

7. Heath, S.; Imregun, M. An improved single-parameter tip-timing method for turbomachinery blade vibration measurements using optical laser probes. Int. J. Mech. Sci. 1996, 38, 1047-1058.

8. Garrido, J.; Dimitriadis, G.; Wright, J.R. A class of methods for the analysis of blade tip timing data from bladed assemblies undergoing simultaneous resonances-Part I: Theoretical development. Int. J. Rotat. Mach. 2007, 2007, 27247.

9. Garrido, J.; Dimitriadis, G.; Wright, J.R. Multiple Frequency Analysis Methods for Blade Tip-Timing Data Analysis. In Proceedings of the ASME 7th Biennial Conference on Engineering Systems Design and Analysis, American Society of Mechanical Engineers, Manchester, UK, 19-22 July 2004. 
10. Zielinski, M.; Ziller, G. Noncontact Crack Detection on Compressor Rotor Blades to Prevent Further Damage after HCF-Failure. Available online: http:/www.mtu.de/en/technologies/ engineering_news/development/Ziller_Noncontact_crack_detection_en.pdf (accessed on 20 January 2014).

11. Kharyton, V.; Laine, J.P.; Thouverez, F. Simulation of tip-timing measurements of a cracked bladed disk forced response. In Proceedings of the ASME Turbo Expo 2010: Power for Land, Sea, and Air, American Society of Mechanical Engineers, Glasgow, UK, 14-18 June 2010.

12. Von Flotow, A.; Mercadal, M.; Tappert, P. Health monitoring and prognostics of blades and disks with blade tip sensors. In Proceedings of the Aerospace Conference, Big Sky, MT, USA, 18-25 March 2000; pp. 433-440.

13. Bendali, S.; Joseph, L.; Marc, B.; Philippe, V.; Charles, B. Modal parameter identification of mistuned bladed disks using tip timing data. J. Sound Vib. 2008, 314, 885-906.

14. Chen, Z.; Yang, Y.; Xie, Y. Non-contact crack detection of high-speed blades based on principal component analysis and Euclidian angles using optical-fiber sensors. Sens. Actuators A Phys. 2013, 201, 66-72.

15. Chen, Z.; Yang, Y.; Bin, G. Blade damage prognosis based on kernel principal component analysis and grey model using subsampled tip-timing signals. Proc. Inst. Mech. Eng. Part C J. Mech. Eng. Sci. 2014, doi:0954406214526584.

16. Ries, S. Reconstruction of real and analytic band-pass signals from a finite number of samples. Signal Proc. 1993, 33, 237-257.

17. Gianluca, R. Design of Blade Tip Timing Measurement Systems Based on Uncertainty Analysis. Available online: http://www.piwg.org/DOC/workshop/58th_IIS_2012_UofPerugia_Rossi.pdf (accessed on 20 January 2014).

18. Beauseroy, P.; Lengellé, R. Nonintrusive turbomachine blade vibration measurement system. Mech. Syst. Signal Proc. 2007, 21, 1717-1738.

19. Garrido, J.; Dimitriadis, G.; Wright, J.R. Blade tip timing measurement of synchronous vibrations of rotating bladed assemblies. Mech. Syst. Signal Proc. 2002, 16, 599-622.

20. Shannon, C.E. Communication in the presence of noise. Proc. IRE 1949, 37, 10-21.

21. Lin, Y.P.; Vaidyanathan, P. Periodically nonuniform sampling of bandpass signals. IEEE Trans. Circuits Syst. II Analog Digit. Signal Proc. 1998, 45, 340-351.

22. Coulson, A.J. A generalization of nonuniform bandpass sampling. IEEE Trans. Signal Proc. 1995, 43, 694-704.

23. Vaughan, R.G.; Scott, L.N.; White, D.R. The theory of bandpass sampling. IEEE Trans. Signal Proc. 1991, 39, 1973-1984.

24. Venkataramani, R.; Bresler, Y. Perfect reconstruction formulas and bounds on aliasing error in sub-Nyquist nonuniform sampling of multiband signals. IEEE Trans. Inf. Theory 2000, 46, 2173-2183.

(C) 2015 by the authors; licensee MDPI, Basel, Switzerland. This article is an open access article distributed under the terms and conditions of the Creative Commons Attribution license (http://creativecommons.org/licenses/by/4.0/). 